Classification

Physics Abstracts

$07.55-06.30 \mathrm{~L}$

\title{
Separation of conductivity and distance measurements for eddy current nondestructive inspection of graphite composite materials
}

\author{
Isabelle Dufour and Dominique Placko \\ Laboratoire d'Electricité, Signaux et Robotique, 61 avenue du Président Wilson, 94235 Cachan \\ Cedex, France
}

(Recelved I June 1992. relised 9 November 1992, accepted 11 January 1993)

\begin{abstract}
This article deals with the study of a process based on the principle of eddy current sensors for the nondestructive evaluation of graphite composite plates. This research has been carried out in the Laboratoire d'Electricité Signaux et Robotique by the team working on datacollecting sensors for robotics in collaboration with Aerospatiale. Eddy current sensors are characterized by their impedance, which varies when a conducting material is approached in their sensitive area. For a given sensor, the output signal depends directly on the electrical and geometrical properties of the object. In the case discussed here, the interesting data are the distance between the sensor and the object, and its local conductivity. In order to invert the relationships between the sensor signal and the properties of the material, an external parametrical model has been developed. A scanning of the surface with a sensor designed for good spatial resolution measurements gives two accurate maps of the useful data.
\end{abstract}

\section{Eddy current sensors principle [1].}

An eddy current sensor is made of an open magnetic core on which an excitation coil is driven by a high frequency sine wave signal. A variable magnetic field is thus created, defining a sensitive area in front of the sensor, which essentially depends on the geometry of the magnetic core. The approach of a target - i.e., a conducting material, which in our case is a graphite composite - into this area modifies the path of the magnetic field (consequently the value of the inductance) and induces losses. The signal is then obtained through the measurement of real and imaginary parts of the winding impedance: it depends on the characteristics of the sensor (geometry, distance between sensor and target, frequency of excitation, etc.) and on the target properties such as conductivity $\sigma$, permeability $\mu$, geometry. In our application, because of the anisotropy of the graphite composite materials, we chose an eddy current sensor which presents a symetry of revolution : a ferrite cup core. 


\section{Measurement and modelling.}

2.1 Normalized IMPEDANCE PLANE [2-4]. - In order to evaluate the sensor efficiency with respect to a given target. we can describe sensor impedance variations in the complex plane : $Z_{\mathrm{c}}=R_{\mathrm{c}}+j X_{\mathrm{c}}$. However, a difficulty in this approach is the growth of the reactance with frequency and the existence of sensor losses when there is no target in front of it $\left(Z_{0}=R_{0}+j X_{0}\right)$. To overcome this difficulty, we prefer to study the variations of normalized impedance $\left(Z_{\mathrm{cn}}=R_{\mathrm{cn}}+j X_{\mathrm{cn}}\right)$, which are only due to the presence of the target. Thus, we can describe the graphic function $X_{\mathrm{cn}}=f\left(R_{\mathrm{cn}}\right)$, where :

$$
R_{\mathrm{cn}}=\frac{R_{\mathrm{c}}-R_{0}}{X_{0}} \quad \text { and } X_{\mathrm{cn}}=\frac{X_{\mathrm{c}}}{X_{0}}
$$

2.2 EXPERIMENTAL RESULTS. - The experimental setup is presented in figure 1. The sensor is placed over a sample of graphite composite, the impedance measurements are made with an impedance analyser (HP 4192A) and the treatment with a computer.

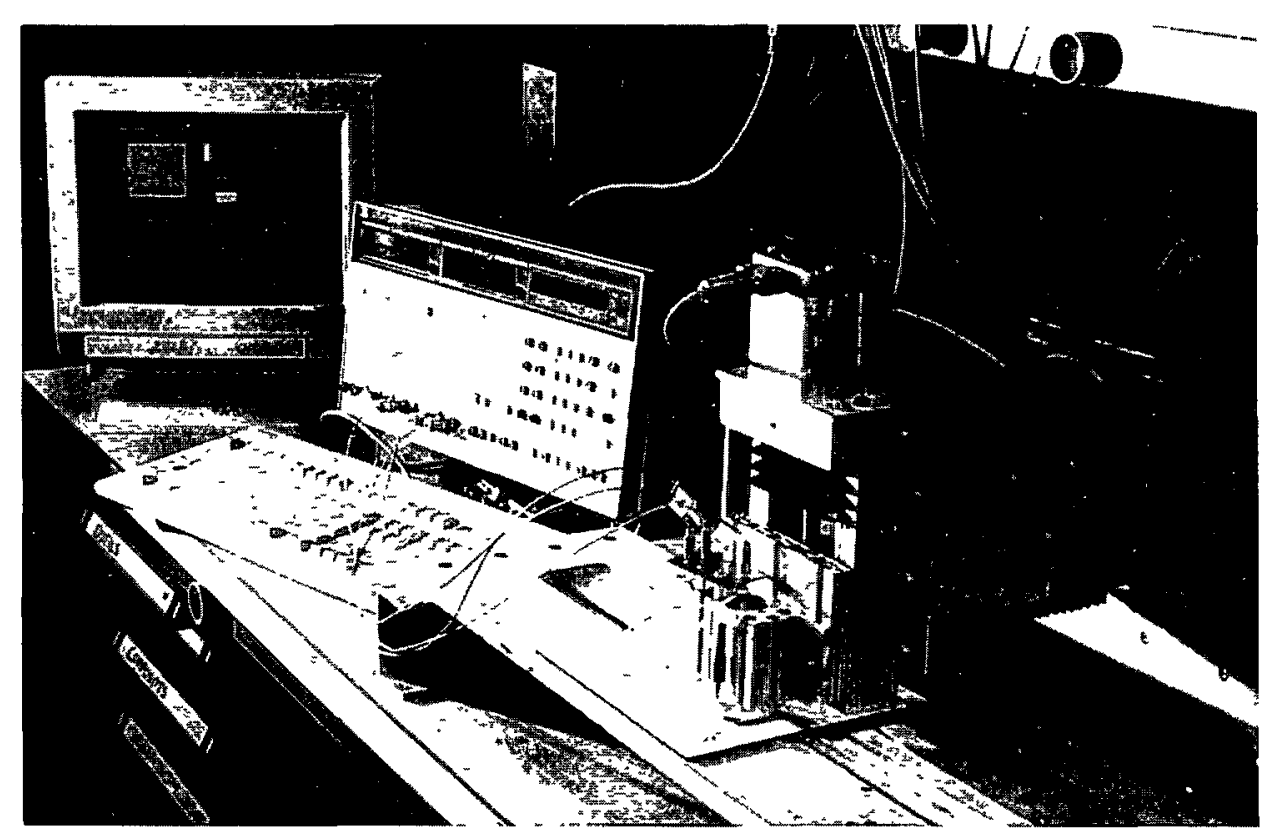

Fig. 1. - Experimental setup.

We present in figure 2 some experimental results obtained for several excitation frequencies and distances between the sensor and a sample of graphite composite placed in front of it. The following remarks may be noted about this figure :

- for a given distance, the curve representing the function $X_{\mathrm{cn}}=f\left(R_{\mathrm{cn}}\right)$ can be assimilated to a circle when the frequency is made to vary. This circle joins the point $(0,1)$ for low frequencies ( $R_{\mathrm{c}}=R_{0}$ and $X_{\mathrm{c}}=X_{0}$ because the eddy currents account for very little) and the 
point $(0, \alpha)$ for very high frequencies (the field does not penetrate the sample : losses in the sample are very small);

- for a given frequency, the curve representing the function $X_{\mathrm{cn}}=f\left(R_{\mathrm{cn}}\right)$ is almost a line passing through the point $(0,1)$ when the distance sample-sensor is made to vary.

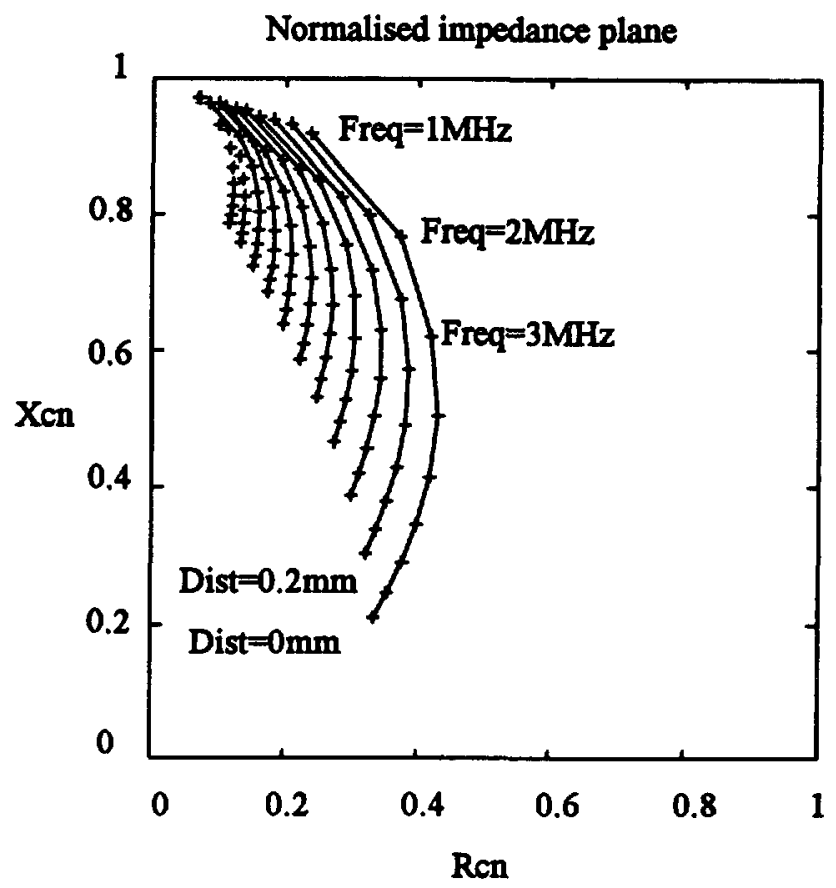

Fig. 2, - Experimental results obtained in the normalized impedance plane with frequency: [1 $\mathrm{MHz}$, $9 \mathrm{MHz}]$ and distance : [0 $\mathrm{mm}, 2 \mathrm{~mm}]$.

Note that measurement points depend on the sensor-target distance and on a factor which is proportional to the product of the excitation frequency by the sample conductivity. In fact, same measurement points should be obtained by increasing the conduictivity of the sample instead of the excitation frequency (in the same proportions). This property readily appears in the Maxwell equations.

2.3 EXTERNAL PARAMETRICAL MODELLING [5]. - An analytical model of the relations between the sensor signal and the properties of the target has been developed. This model is based on an electrical diagram constituted by a transformer in which the primary and the secondary circuits respectively symbolize the sensor and the target (Fig. 3). The load impedance is linked to the complex power dissipated in the target. A very simple interpretation is that the real impedance represents the resistance of the loop in which eddy currents are induced ( $\operatorname{Re}$ will be consequently proportional to $1 / \sigma$ ), and the imaginary term accounts for the leakage inductance of this circuit. The coupling coefficient $k$, is linked to the distance between the sensor and the target : it decreases when the distance increases. These parameters are obtained by identification with experimental results.

When there is no target in front of the sensor - i.e., the coupling factor $k$ is zero - the impedance of the transformer is $Z_{0}=R_{0}+j X_{0}$. In the presence of target, this impedance 


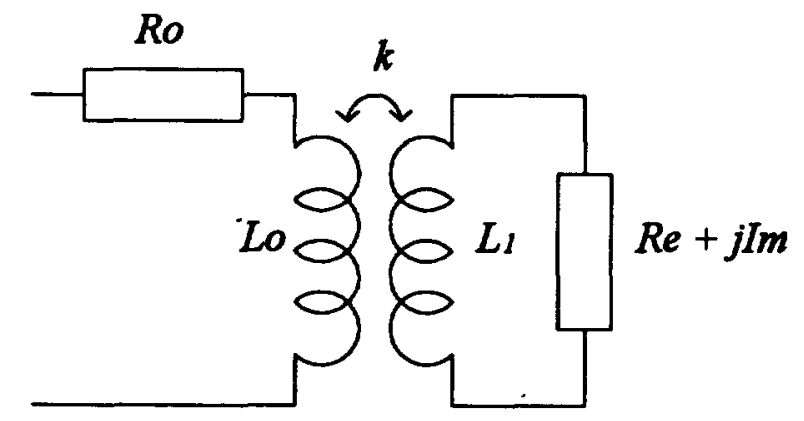

Fig. 3. - External parametrical model.

becomes $Z_{c}$, as described by the following equation :

$$
Z_{\mathrm{c}}=R_{0}+j L_{0} \omega+\frac{k^{2} L_{0} L_{1} \omega^{2}}{\operatorname{Re}+j L_{1} \omega+j \mathrm{Im}}
$$

So, obtaining :

$$
R_{\mathrm{cn}}=\frac{k^{2} L_{1} \omega \mathrm{Re}}{\operatorname{Re}^{2}+\left(L_{1} \omega+\mathrm{Im}\right)^{2}}
$$

and

$$
X_{\mathrm{cn}}=1-\frac{k^{2} L_{1} \omega\left(L_{1} \omega+\mathrm{Im}\right)}{\operatorname{Re}^{2}+\left(L_{1} \omega+\mathrm{Im}\right)^{2}}
$$

we get :

$$
\left(R_{\mathrm{cn}}+\frac{k^{2} \mathrm{Im}}{2 \mathrm{Re}}\right)^{2}+\left(X_{\mathrm{cn}}-1+\frac{k^{2}}{2}\right)^{2}=\frac{k^{4}}{4}\left(\frac{\mathrm{Im}^{2}}{\mathrm{Re}^{2}}+1\right)
$$

and

$$
X_{\mathrm{cn}}=1-\frac{L_{1} \omega+\mathrm{Im}}{\operatorname{Re}} R_{\mathrm{cn}}
$$

- On the assumption that $\operatorname{Im}, \operatorname{Re}$ and $L_{1}$ do not depend (for a given sample) on the distance between the sensor and the target, we obtain a line for a given frequency in the normalized impedance plane when the coupling coefficient changes. The slope of the line is $\left(L_{1} \omega+\mathrm{Im}\right) / \mathrm{Re}$. Note that, if Im is negligible in respect to $L_{1} \omega$ or proportional to $\omega$, we find again that the slope is proportional to the product frequency conductivity (Fig. 4).

- On the assumption that $\mathrm{Im} / \mathrm{Re}$ is constant (for a given sample), we obtain a circle in the normalised impedance plane for a given coupling coefficient $k$, when the frequency changes. The centres of circles are on the line connecting $(0,1)$ and $(-\operatorname{Im} / \operatorname{Re}, 0)$. Moreover, these circles intercept the ordinate axis at $\left(0,1-k^{2}\right)$ (Fig. 4).

It can be seen that this electrical model correlates sufficiently with the experiments. Therefore, we can discuss identification of parameters.

2.4 IDENTIFICATION. - For a given distance, we identify the curve obtained in the normalized impedance plane to a circle with the least square method. By means of this circle equation, we get the values of the ratio $\mathrm{Im} / \mathrm{Re}$ and of the coupling coefficient $k$. 


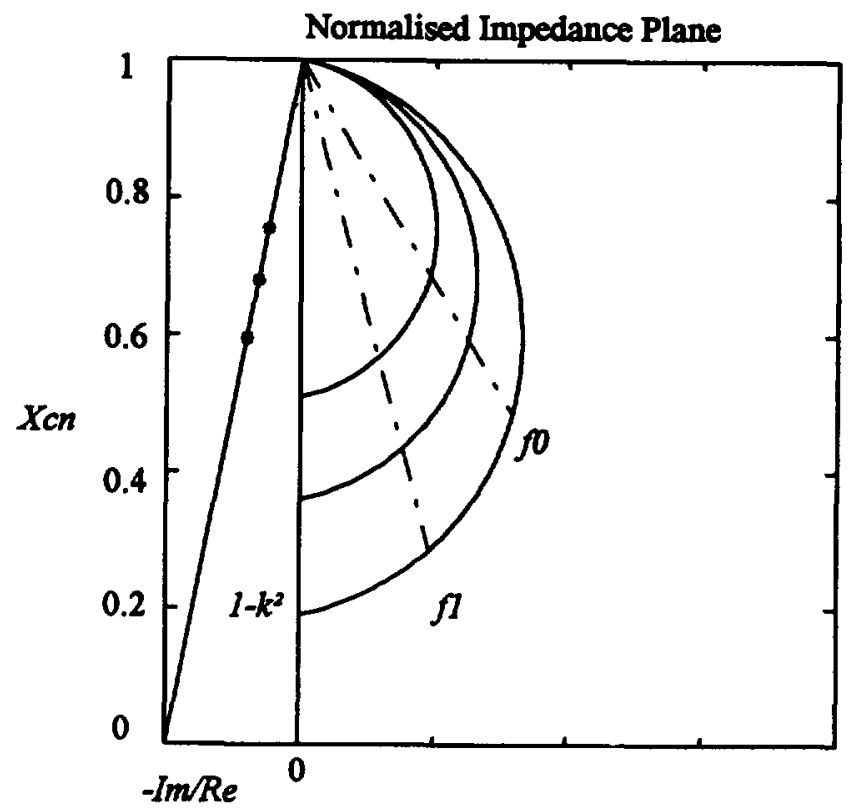

$\operatorname{Ren}$

Fig. 4. - Normalized impedance plane analysis when model parameters are constant.

Results are given in figures $5 a$ and $5 b$ for two different ranges of frequencies. We see that the ratio $\mathrm{Im} / \mathrm{Re}$ is not constant but increases with frequency, and depends on coupling coefficient. We find out that it varies linearly versus $1-k^{2}$.
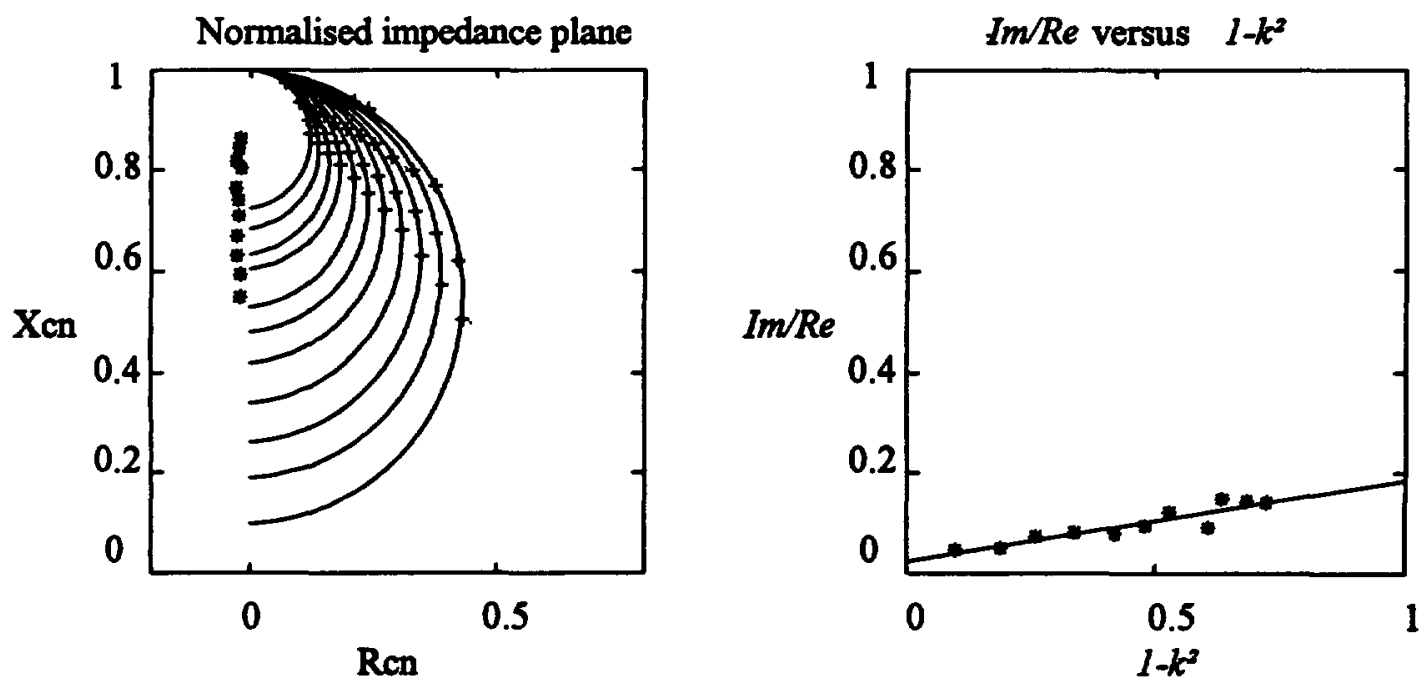

a)

Fig. 5. - a) Identification results with frequency [1 $\mathrm{MHz}, 4 \mathrm{MHz}$ ] and distance [0 mm, $2 \mathrm{~mm}$ ]. b) Identification results with frequency [5 MHz, $9 \mathrm{MHz}]$ and distance [0 mm, $2 \mathrm{~mm}$. c) Identification results with frequency $[1 \mathrm{MHz}, 9 \mathrm{MHz}]$ and distance $[0 \mathrm{~mm}, 2 \mathrm{~mm}]$. 

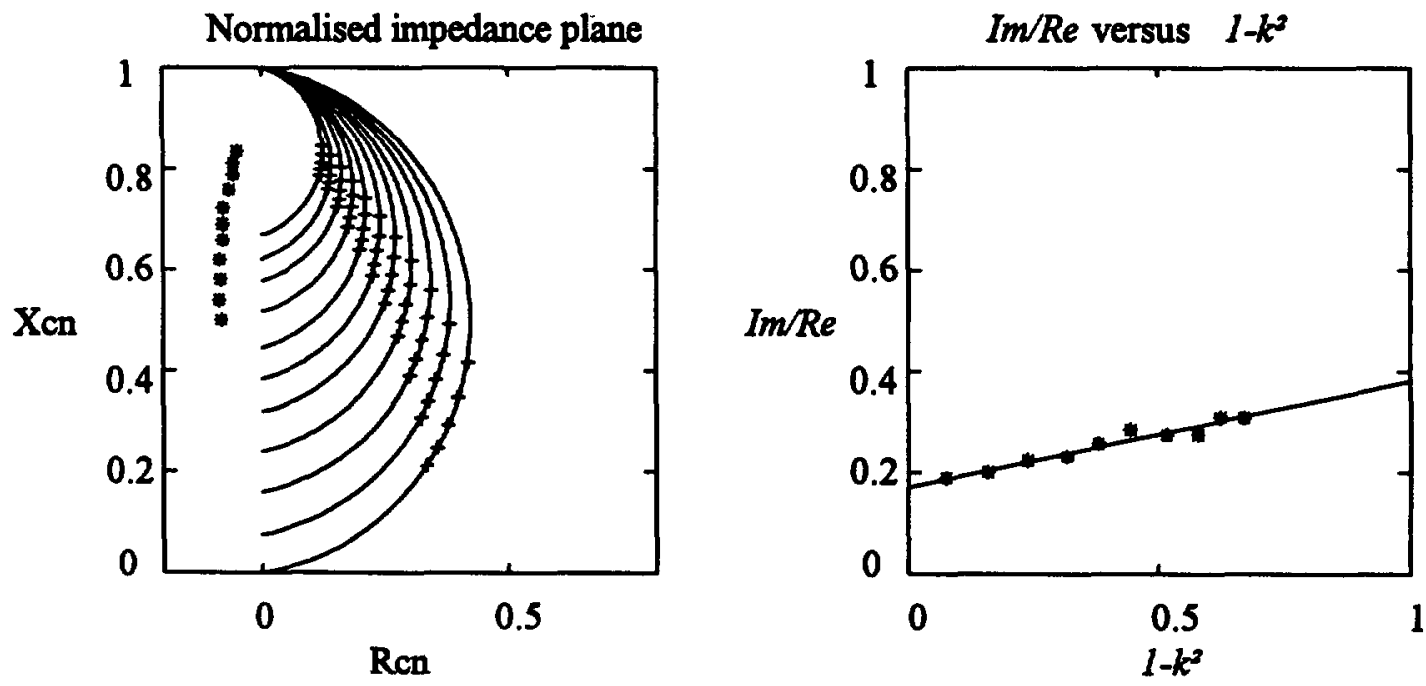

b)
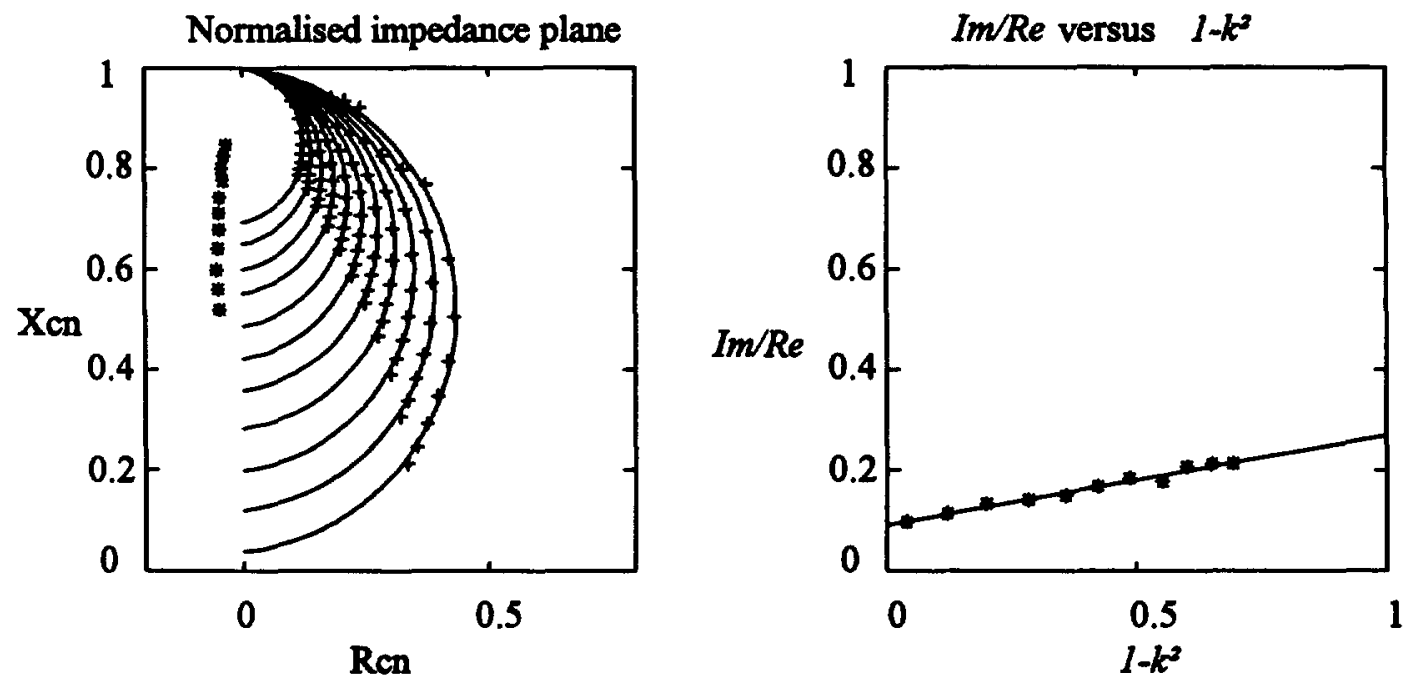

c)

Fig. 5 (continued).

Figure $5 \mathrm{c}$ represents the results obtained for the whole range of frequency. Note that goods results are obtained even considering that $\mathrm{Im} / \mathrm{Re}$ does not vary with frequency; the model is therefore much simpler!

\section{Model inversion and results.}

3.1 MODEL INVERSION. - The user of a nondestructive testing device is more interested by physical properties of the object studied than by electrical properties of the signal sensor. By the use of our model, we can estimate from both parts of the sensor impedance two independent data concerning the object placed in front of the sensor: the distance between the sensor and the target, and its local conductivity. 
Distance estimation. - We can estimate the sensor-target distance in calculating the value $\alpha=1-k^{2}$ defined as the ordinate of the intersection point of the circle and the ordinate axis (Fig. 6). This point corresponds to the simpler case of use of eddy current sensors (there is no losses and the magnetic field is tangent to the surface of the target). This case can be studied with the electrical-image method which gives in some particular geometries of sensors an analytical relation connecting $\alpha$ to the distance [1].

Conductivity estimation. - As it can be seen previously, conductivity is coupled to the slope of the line obtained when $k$ varies. It is simpler to boil down to the case of $k=1$; the parametrization of this circle towards $\sigma$ can be effected by introducing the angle $\theta$ (Fig. 6).

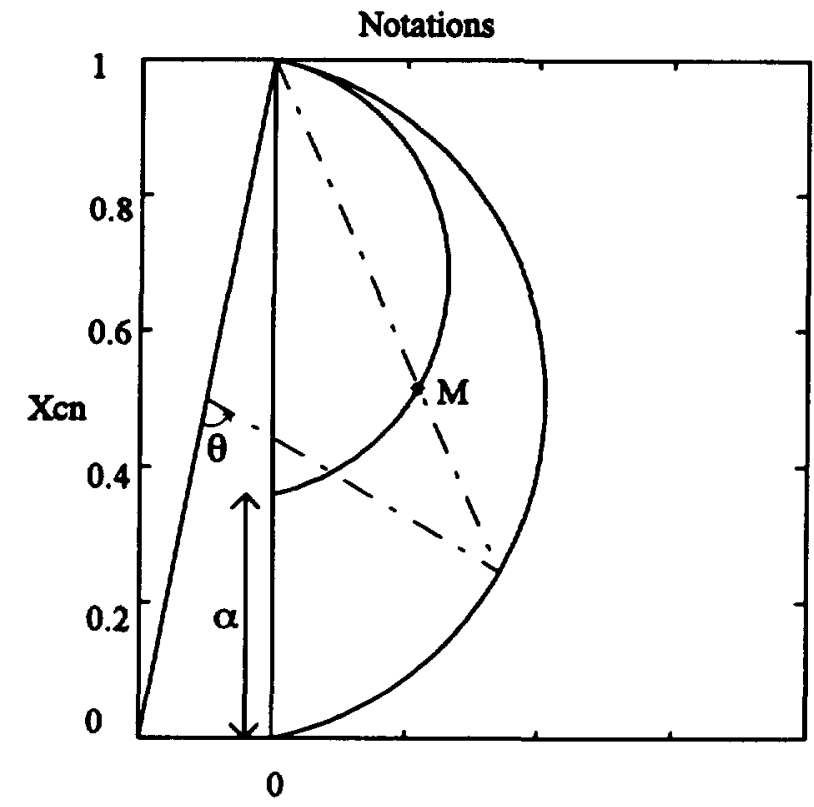

\section{$\operatorname{Ren}$}

Fig. 6. - Definition of alpha and theta used for the model inversion.

Since $\theta$ decreases more rapidly when frequency is low than high, it is more interesting to study variations of $1 / \theta$ versus frequency in order to improve the linearity of the curve (Fig. 7).

In order to obtain the values of distance and conductivity, we made a first order development of $\alpha$ and $1 / \theta$ about the point defined by freq $=f_{0}$, dist $=d_{0}$ and $\sigma=\sigma_{0}$. Thus, we get the following equations :

$$
\alpha=\alpha_{0}+\alpha_{1} \cdot \text { dist and } 1 / \theta=a_{0}+a_{1} \text {. freq } .
$$

Thus the distance value is readily calculable :

$$
\operatorname{dist}=\frac{\alpha-\alpha_{0}}{\alpha_{1}}
$$



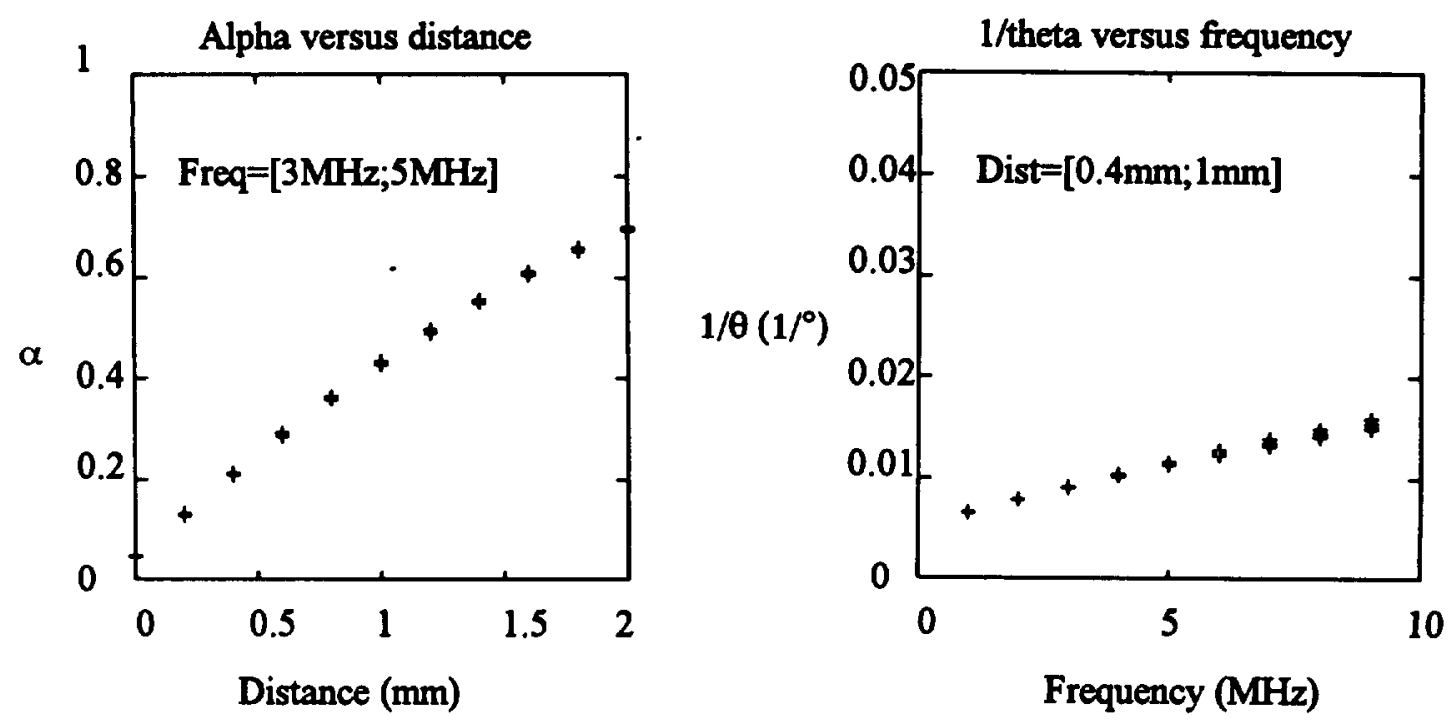

Fig. 7. - Graphs representing the relationships between alpha and distance and between theta and frequency.

Taking into account that $\theta$ is a function of the product frequency conductivity, we can calculate the relative variation of conductivity :

$$
\frac{\sigma-\sigma_{0}}{\sigma_{0}}=\frac{1 / \theta-a_{0}-f_{0} \cdot a_{1}}{a_{1} \cdot f_{0}}
$$

3.2 RESULTS. - Experimental tests have been performed by scanning a sensor over a graphite composite plate which has been impacted. In order to test the method, the plate has been voluntary tilted. The results of this scanning are presented below (Fig. 8) : the raw information (real and imaginary parts of the sensor signal) is coded according to a colour scale (the whole range of variations is coded linearly with 16 colours). We can see the drastic effects of a small distance variation (about $1 \mathrm{~mm}$ ) on the normalized impedance.

Our inversion method applied to the previous signals gives the results presented below (Fig. 9). Useful information is now, respectively, the distance between the sensor and the sample, and the local conductivity for each measurement point. With this excitation frequency ( $4 \mathrm{MHz}$ ), the skin depth is about $2.5 \mathrm{~mm}$, then the local conductivity estimated concerns the whole thickness of the sample.

Owing to this method, we can simultaneously know if the impact has affected the surface of the sample and estimate the internal extent of the material deterioration. The degree of gravity can be appreciated by studying the geometrical extensions and the conductivity variation range.

\section{Conclusion.}

This signal processing allows us to know simultaneously the distance sensor-target and the local target conductivity: the main interest of such a method is to have a control over conductivity independent of the parasitic height variations during the inspection. This process can be used in the nondestructive inspection of graphite composite materials for impact 

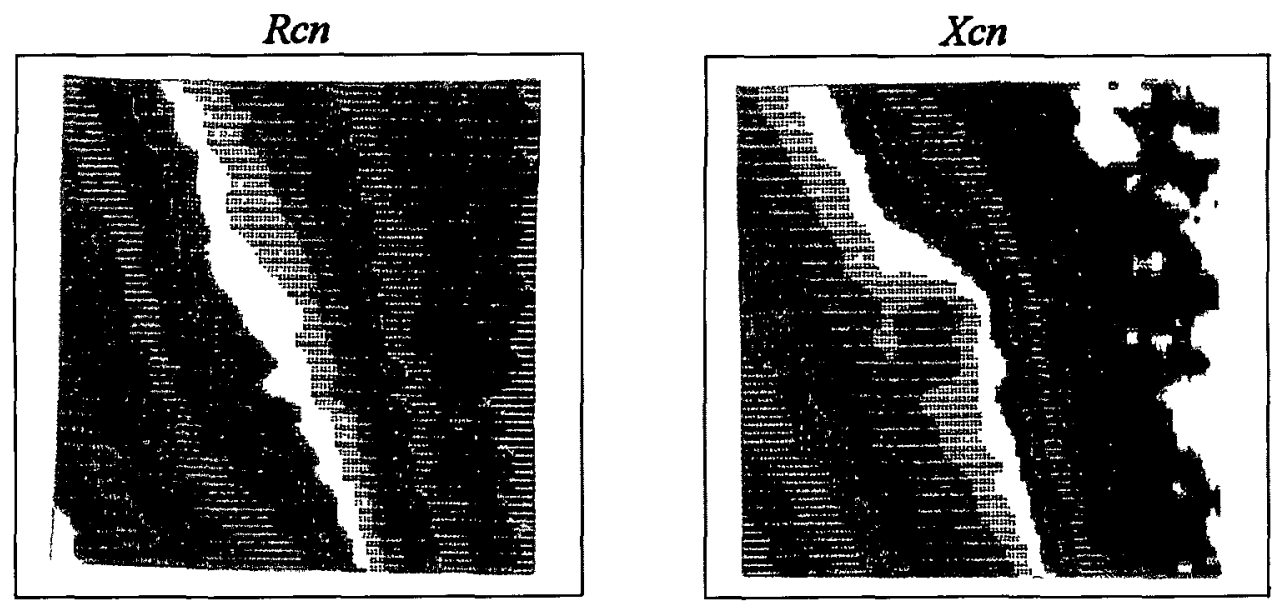

Fig. 8. - Maps of $R_{\mathrm{cn}}$ and $X_{\mathrm{cn}}$ obtained on a graphite composite sample with impact, frequency $=4 \mathrm{MHz}$.

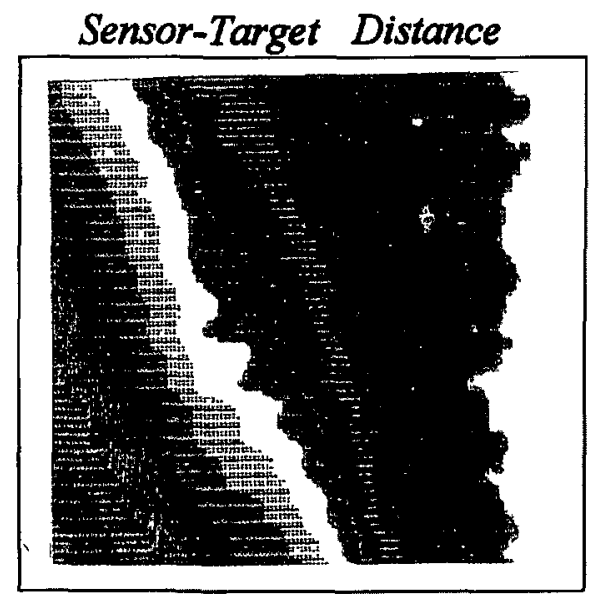

Minimum: $1.67 \mathrm{~mm}$

Maximum: $2.95 \mathrm{~mm}$

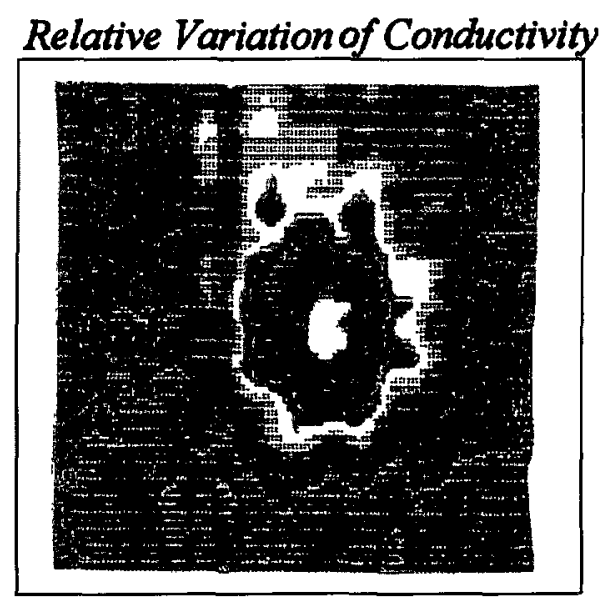

Minimum: $-6.2 \%$

Maximum: $+8.9 \%$

Fig. 9. - Maps of the distance and relative variation of the local conductivity of the graphite composite sample with impact, frequency $=4 \mathrm{MHz}$.

detection for in-service materials or for the detection of fibre ratio variations after the composite making. It should be noted that the method application area is greater than graphite composite materials : this treatment can be applied to other conducting samples, like metallic samples, provided that the frequency is changed. Some extensions of this model can be obtained by taking into account both dependence of Im/Re versus distance and conductivity (or frequency) for large ranges of conductivity measurements, or for systems with a variable or multi excitation frequency (multifrequency could be used for estimate the local conductivity versus the depth). 


\section{References}

[1] PlACKo D., Contribution à la conception de capteurs inductifs pour la robotique industrielle, Rapport de synthèse pour l'habilitation à diriger des recherches en sciences (22 mai 1990).

[2] Hagemaier D. J., Eddy current impedance plane analysis, Mater. Eval. 41 (1983) 211-218.

[3] Vernon S., The universal impedance diagram of ferrite pot core eddy current transducer, IEEE Trans. Magn. 25, n 3 (may 1989).

[4] VAlleaU A. R., Eddy current non destructive testing of graphite composite materials, Mater. Eval. 48 (1990) 230-239.

[5] Placko D., Santander E., Capteur de mesure de faibles épaisseurs à courants de Foucault, Congrès MESUCORA 91, Paris, session n 15, pp. 3-17. 